\title{
Turnaround radius in scalar-tensor gravity with quasilocal mass
}

\author{
Valerio Faraon: and Andrea Giust \\ Department of Physics and Astronomy, Bishop's University, Sherbrooke, Québec, Canada J1M 1Z7 \\ Jeremy Côt团 \\ Perimeter Institute for Theoretical Physics, 31 Caroline Street North, Waterloo, ON N2L 2Y5, Canada
}

\begin{abstract}
Following an existing procedure in general relativity, the turnaround radius of a spherical structure is studied in scalar-tensor gravity using a new prescription for the analog of the Hawking-Hayward quasilocal mass in this class of theories.
\end{abstract}

\section{INTRODUCTION}

The fact that today we live in an accelerated epoch of the history of the universe [1, 2] has several consequences. Among these is that there exists an upper limit to the size of cosmic structures that are bound. Structures with this size, or larger, are dispersed by the cosmic acceleration, while smaller structures can remain bound [3]-[8]. If the structure is spherical, the radius at which the local gravitational attraction balances exactly the cosmological expansion is known as the turnaround radius [3]- [8] .

Turnaround physics has been proposed as a probe of dark energy scenarios [3]-[8]. It was soon realized that the turnaround radius and turnaround physics are also potential probes of the theory of gravity [9]-[15]. Spherically symmetric situations have received most of the attention in the literature thus far but, recently, more realistic nonspherical situations have been discussed [16-21]. In fact, the observational search for the turnaround radius suffers from extrapolating the theory developed in oversimplified spherically symmetric scenarios to realistic non-spherical ones 22 25].

In the presence of spherical symmetry, the turnaround radius is usually defined (both in general relativity (GR) and in alternative gravity) using radial timelike geodesics and imposing the condition of zero radial acceleration at the turnaround radius. This procedure is, unfortunately, gauge-dependent. In GR, an alternative definition uses the Hawking-Hayward quasilocal mass [26, 27]. In the case of spherical symmetry, to which we restrict ourselves in this work, the Hawking-Hayward quasilocal energy reduces [28] to the better known Misner-Sharp-Hernandez mass 29]. In general, the Hawking-Hayward quasilocal mass of a cosmological structure described by a perturbation of a Friedmann-Lemaître-Robertson-Walker (FLRW) universe splits, in a gauge-invariant way, into a "local" and a "cosmological" contribution [30 32] (the same splitting of the Hawking-Hayward quasilocal mass has been applied to study Newtonian dust perturbations in the matter era [31] and gravitational lensing by the cosmological constant or by dark energy [33, 34]). When these two contributions are equal (in absolute value), the turnaround radius is achieved [35]. In a universe dominated by dark energy with equation of state $P_{D E} \simeq-\rho_{D E}$, the numerical value of the turnaround radius of a spherical structure obtained with this definition is very close to the one obtained with radial timelike geodesics 35 .

When trying to generalize the quasilocal definition of turnaround radius to scalar-tensor gravity, the first difficulty that one encounters consists of generalizing the Hawking-Hayward quasilocal energy to this class of theories. A few tentative definitions have been given in the literature for different spacetime geometries or for special situations [36], and they disagree with each other. However, one recent proposal relies on minimal assumptions, in the sense that it is based simply on writing the scalar-tensor field equations as effective Einstein equations, with terms containing the Brans-Dicke-like field and its derivatives forming an effective energy-momentum tensor [37]. This procedure is very common in scalar-tensor gravity, has been very useful in several contexts (e.g., 38, 39]), and produces an unambiguous prescription for the scalar-tensor analog of the Hawking-Hayward quasilocal mass, which we adopt here.

We apply this new generalization of the Hawking-Hayward mass in scalar-tensor gravity to the problem of the turnaround radius in cosmology. Restricting ourselves to spherical symmetry for simplicity, we use the gauge-invariant procedure proposed in Ref. [35] in the context of GR and we characterize the turnaround radius in scalar-tensor gravity. We will begin in the context of a perturbed FLRW universe in the conformal Newtonian gauge. 
To fix the notation, we recall the basic equations of scalar-tensor gravity. We follow the conventions of Ref. [40]: the metric signature is -+++ and we use units in which the speed of light and Newton's constant are unity. The (Jordan frame) scalar-tensor action is

$$
S_{S T}=\int d^{4} x \sqrt{-g}\left\{\left[\frac{1}{16 \pi}\left(\phi \mathcal{R}-\frac{\omega(\phi)}{\phi} g^{a b} \nabla_{a} \phi \nabla_{b} \phi\right)-V(\phi)\right]+\mathcal{L}_{(m)}\right\},
$$

where $\mathcal{R}$ is the Ricci scalar of the metric $g_{a b}$, which has determinant $g$, while $\phi$ is the Brans-Dicke-like scalar field and, approximately, the inverse of the spacetime-dependent effective gravitational coupling $G_{\text {eff }}$. $V(\phi)$ is the potential of this scalar field, while $\mathcal{L}^{(m)}$ is the Lagrangian density of matter. The field equations read

$$
\begin{aligned}
& R_{a b}-\frac{1}{2} g_{a b} \mathcal{R}=\frac{8 \pi}{\phi} T_{a b}+\frac{\omega}{\phi^{2}}\left(\nabla_{a} \phi \nabla_{b} \phi-\frac{1}{2} g_{a b} \nabla^{c} \phi \nabla_{c} \phi\right)+\frac{1}{\phi}\left(\nabla_{a} \nabla_{b} \phi-g_{a b} \square \phi\right)-\frac{V}{2 \phi} g_{a b}, \\
& \square \phi=\frac{1}{2 \omega+3}\left(8 \pi T-\frac{d \omega}{d \phi} \nabla^{c} \phi \nabla_{c} \phi+\phi \frac{d V}{d \phi}-2 V\right),
\end{aligned}
$$

where $T_{a b}=-\frac{2}{\sqrt{-g}} \frac{\delta}{\delta g^{a b}}\left(\sqrt{-g} \mathcal{L}_{(m)}\right)$ is the matter energy-momentum tensor with trace $T \equiv T^{a}{ }_{a} . f(\mathcal{R})$ theories of gravity [41] are a subclass of scalar-tensor theories described by the action

$$
S=\int d^{4} x \sqrt{-g} f(\mathcal{R})+S^{(m)}
$$

where $f(\mathcal{R})$ is a nonlinear function of the Ricci scalar. The scalar degree of freedom is $\phi=f^{\prime}(\mathcal{R})$ and the scalar field potential is given implicitly by

$$
V(\phi)=\phi \mathcal{R}(\phi)-\left.f(\mathcal{R}(\phi))\right|_{\phi=f^{\prime}(\mathcal{R})}
$$

in these theories. This action turns out to be equivalent to the scalar-tensor one [41]

$$
S=\int d^{4} x \frac{\sqrt{-g}}{16 \pi}[\phi \mathcal{R}-V(\phi)]+S^{(m)} .
$$

This is a Brans-Dicke action with vanishing Brans-Dicke parameter $\omega$ and a rather contrived potential $V$ for the Brans-Dicke scalar $\phi$.

\section{TURNAROUND RADIUS WITH QUASILOCAL MASS IN SCALAR-TENSOR GRAVITY}

As usual in the literature on the turnaround radius (and on structure formation), one does not include vector and tensor perturbations in the perturbed FLRW metric, which is justified at lower order for the non-relativistic velocities of the matter perturbations. The perturbed FLRW metric and Brans-Dicke-like scalar field are

$$
\begin{gathered}
d s^{2}=a^{2}(\eta)\left[-(1+2 \psi) d \eta^{2}+(1-2 \Phi)\left(d r^{2}+r^{2} d \Omega_{(2)}^{2}\right)\right] \\
=g_{\mu \nu} d x^{\mu} d x^{\nu}=I_{\mu \nu} d x^{\mu} d x^{\nu}+h_{\mu \nu} d x^{\mu} d x^{\nu} \\
\phi(\eta, r)=\phi_{0}(\eta)+\delta \phi(r)
\end{gathered}
$$

where $R^{2} d \Omega_{(2)}^{2}=h_{\mu \nu} d x^{\mu} d x^{\nu}=R^{2}\left(d \theta^{2}+\sin ^{2} \theta d \varphi^{2}\right)$ is the line element on the 2-spheres of symmetry with areal radius

$$
R(\eta, r)=a(\eta) r \sqrt{1-2 \Phi} \approx a(\eta) r(1-\Phi) \equiv R_{0}(1-\Phi)
$$

to first order, and

$$
\mathcal{O}\left(\frac{\delta \phi}{\phi_{0}}\right)=\mathcal{O}(\Phi)=\mathcal{O}(\psi)
$$


The tensors $I_{a b}$ and $h_{a b}$ form the metric $g_{a b}$. In particular, the non-zero components $I_{\mu \nu}$ are 0,1 (the time and radial components), while the non-zero components $h_{\mu \nu}$ are 2,3 (the angular ones). This simply provides a convenient splitting of the metric for later on.

We also assume that the metric and scalar field perturbations do not depend on time,

$$
\partial_{\eta} \Phi=\partial_{\eta} \psi=\partial_{\eta} \delta \phi=0 \text {. }
$$

This assumption is fine for structures of size much smaller than the Hubble radius $H^{-1}$, but it would not be justified for inflationary perturbations that cross outside the horizon in the early universe. ${ }^{1}$ We have in mind applications to the turnaround radius of cosmic structures in the late universe. The structures of interest are of the size of galaxy groups or clusters, therefore, much smaller than the Hubble radius and they evolve on time scales much smaller than the Hubble time. For these structures, it is justifiable to neglect the time dependence in the metric potentials (and, accordingly, in the Brans-Dicke scalar perturbation).

Explicitly, we have (again, keep in mind the range of the indices and that we are computing this to first order)

$$
\begin{aligned}
& I_{\mu \nu}=\left(\begin{array}{cccc}
-(1+2 \psi) a^{2} & 0 & 0 & 0 \\
0 & (1-2 \Phi) a^{2} & 0 & 0 \\
0 & 0 & 0 & 0 \\
0 & 0 & 0 & 0
\end{array}\right) \\
& =I_{\mu \nu}^{(0)}+\delta I_{\mu \nu} \\
& =\left(\begin{array}{cccc}
-a^{2} & 0 & 0 & 0 \\
0 & a^{2} & 0 & 0 \\
0 & 0 & 0 & 0 \\
0 & 0 & 0
\end{array}\right)+\left(\begin{array}{cccc}
-2 \psi a^{2} & 0 & 0 & \\
0 & -2 \Phi a^{2} & 0 & 0 \\
0 & 0 & 0 & 0 \\
0 & 0 & 0 & 0
\end{array}\right), \\
& I^{\mu \nu}=\left(\begin{array}{cccc}
0 & 0 & -\frac{(1-2 \Psi)}{a^{2}} & 0 \\
0 & 0 & 0 & \frac{(1+2 \Phi)}{a^{2}} \\
0 & 0 & 0 & 0 \\
0 & 0 & 0 & 0
\end{array}\right)=I_{(0)}^{\mu \nu}+\delta I^{\mu \nu} \\
& =\left(\begin{array}{cccc}
-\frac{1}{a^{2}} & 0 & 0 & 0 \\
0 & \frac{1}{a^{2}} & 0 & 0 \\
0 & 0 & 0 & 0 \\
0 & 0 & 0 & 0
\end{array}\right)+\left(\begin{array}{cccc}
\frac{2 \psi}{a^{2}} & 0 & 0 & 0 \\
0 & \frac{2 \Phi}{a^{2}} & 0 & 0 \\
0 & 0 & 0 & 0 \\
0 & 0 & 0 & 0
\end{array}\right) \\
& h_{\mu \nu}=\left(\begin{array}{cccc}
0 & 0 & 0 & 0 \\
0 & 0 & 0 & 0 \\
0 & 0 & a^{2}(1-2 \Phi) r^{2} & 0 \\
0 & 0 & 0 & a^{2}(1-2 \Phi) r^{2} \sin ^{2} \theta
\end{array}\right) \\
& =h_{\mu \nu}^{(0)}+\delta h_{\mu \nu}=(1-2 \Phi) h_{\mu \nu}^{(0)},
\end{aligned}
$$

${ }^{1}$ We thank Enea Romano for bringing this point to our attention. 


$$
\begin{aligned}
h^{\mu \nu} & =\left(\begin{array}{cccc}
0 & 0 & 0 & 0 \\
0 & 0 & 0 & 0 \\
0 & 0 & \frac{(1+2 \Phi)}{a^{2} r^{2}} & 0 \\
0 & 0 & 0 & \frac{(1+2 \Phi)}{a^{2} r^{2} \sin ^{2} \theta}
\end{array}\right) \\
& =h_{(0)}^{\mu \nu}+\delta h^{\mu \nu}=(1+2 \Phi) h_{(0)}^{\mu \nu} .
\end{aligned}
$$

The quasilocal mass contained in a 2-sphere of areal radius $R$ in scalar-tensor theory, in spherical symmetry, is (cf. Eq. (20) of Ref. [37])

$$
\begin{aligned}
M_{S T}= & \frac{\phi R^{2}}{4}\left[h^{a c} h^{b c} C_{a b c d}+\frac{8 \pi}{\phi} h^{a b} T_{a b}-\frac{16 \pi T}{3 \phi}+\frac{\omega}{\phi^{2}}\left(h^{a b} \nabla_{a} \phi \nabla_{b} \phi-\frac{1}{3} g^{a b} \nabla_{a} \phi \nabla_{b} \phi\right)\right. \\
& \left.+\frac{h^{a b} \nabla_{a} \nabla_{b} \phi}{\phi}+\frac{V}{3 \phi}\right],
\end{aligned}
$$

where $C_{a b c d}$ is the Weyl tensor and $T \equiv g^{a b} T_{a b}$ is the trace of the matter energy-momentum tensor.

Let us compute the various terms in the right hand side of Eq. (17) separately. The first term is

$$
\begin{aligned}
h^{a c} h^{b c} C_{a b c d} & =h^{22}\left(h^{22} C_{2222}+h^{33} C_{2323}\right)+h^{33}\left(h^{22} C_{3232}+h^{33} C_{3333}\right) \\
& =2 h_{(0)}^{22} h_{(0)}^{33} C_{2323}=\frac{2 C_{2323}}{a^{4} r^{4} \sin ^{2} \theta},
\end{aligned}
$$

where the Weyl tensor is of first order in the perturbations since it vanishes exactly in the FLRW background. Computing the component $C_{2323}$ gives

$$
h^{a c} h^{b c} C_{a b c d}=\frac{2}{3 r a^{2}}\left(\psi^{\prime}+\Phi^{\prime}-r \psi^{\prime \prime}-r \Phi^{\prime \prime}\right)
$$

where a prime denotes differentiation with respect to the comoving radius $r$.

The background fluid consists of dark energy and a dust, with total energy density and pressure

$$
\rho_{(0)}=\rho_{(0)}^{(D E)}+\rho_{(0)}^{(d u s t)}, \quad P_{(0)}=P_{(0)}^{(D E)} .
$$

Accordingly, the matter energy-momentum tensor is decomposed as

$$
\begin{aligned}
T_{a b}= & (P+\rho) u_{a} u_{b}+P g_{a b}=\left(P_{(0)}+\delta P+\rho_{(0)}+\delta \rho\right)\left(u_{a}^{(0)}+\delta u_{a}\right)\left(u_{b}^{(0)}+\delta u_{b}\right)+\left(P_{(0)}+\delta P\right)\left(g_{a b}^{(0)}+\delta g_{a b}\right) \\
= & \left(P_{(0)}+\rho_{(0)}\right) u_{a}^{(0)} u_{b}^{(0)}+P_{(0)} g_{a b}^{(0)}+(\delta P+\delta \rho) u_{a}^{(0)} u_{b}^{(0)}+\left(\rho_{(0)}+P_{(0)}\right)\left(u_{a}^{(0)} \delta u_{b}+u_{b}^{(0)} \delta u_{a}\right) \\
& +P_{(0)} \delta g_{a b}+\delta P g_{a b}^{(0)} \\
\equiv & T_{a b}^{(0)}+\delta T_{a b} .
\end{aligned}
$$

The non-vanishing components of the matter energy-momentum tensor are

$$
\begin{aligned}
& T_{00}^{(0)}=\rho_{(0)} a^{2}, \\
& T_{11}^{(0)}=a^{2} P_{(0)}, \\
& T_{22}^{(0)}=a^{2} P_{(0)} r^{2}, \\
& T_{33}^{(0)}=a^{2} P_{(0)} r^{2} \sin ^{2} \theta,
\end{aligned}
$$


and

$$
\begin{aligned}
& \delta T_{00}=-2 a^{2}\left(\rho_{(0)}+2 P_{(0)}\right) \psi+a^{2} \delta \rho, \\
& \delta T_{11}=a^{2} P_{(0)}\left(\frac{\delta P}{P_{(0)}}-2 \Phi\right), \\
& \delta T_{22}=a^{2} r^{2} P_{(0)}\left(\frac{\delta P}{P_{(0)}}-2 \Phi\right), \\
& \delta T_{33}=a^{2} r^{2} \sin ^{2} \theta P_{(0)}\left(\frac{\delta P}{P_{(0)}}-2 \Phi\right),
\end{aligned}
$$

so we obtain

$$
8 \pi h^{a b} T_{a b}=16 \pi P_{(0)}\left(1+\frac{\delta P}{P_{(0)}}\right) .
$$

Then the trace of the energy-momentum tensor is

$$
\begin{aligned}
-\frac{16 \pi T}{3} & =-\frac{16 \pi}{3}\left(I_{(0)}^{a b}+\delta I^{a b}+h_{(0)}^{a b}+\delta h^{a b}\right)\left(T_{a b}^{(0)}+\delta T_{a b}\right) \\
& =-\frac{16 \pi}{3}\left(T^{(0)}+\delta T\right),
\end{aligned}
$$

where

$$
T^{(0)}=g_{(0)}^{a b} T_{a b}^{(0)}=-\rho_{(0)}+3 P_{(0)}
$$

and

$$
\begin{aligned}
\delta T & =I_{(0)}^{a b} \delta T_{a b}+\delta I^{a b} T_{a b}^{(0)}+h_{(0)}^{a b} \delta T_{a b}+\delta h^{a b} T_{a b}^{(0)} \\
& =4\left(\rho_{(0)}+P_{(0)}\right) \psi-\delta \rho+3 \delta P+2 P_{(0)} \Phi
\end{aligned}
$$

so that

$$
\begin{aligned}
-\frac{16 \pi T}{3}= & -\frac{16 \pi}{3}\left[-\rho_{(0)}+3 P_{(0)}+4\left(\rho_{(0)}+P_{(0)}\right) \psi\right. \\
& \left.-\delta \rho+3 \delta P+2 P_{(0)} \Phi\right] .
\end{aligned}
$$

The next term for $M_{S T}$ is

$$
h^{a b} \nabla_{a} \phi \nabla_{b} \phi=h^{22}\left(\partial_{2} \phi\right)^{2}+h^{33}\left(\partial_{3} \phi\right)^{2}=0
$$

and, to first order in the perturbations (we expand $\omega$ around the background field $\phi_{0}$ ),

$$
\frac{\omega}{\phi}\left(h^{a b} \nabla_{a} \phi \nabla_{b} \phi-\frac{1}{3} g^{a b} \nabla_{a} \phi \nabla_{b} \phi\right)=\frac{\left(\omega_{0}-2 \omega_{0} \psi+d \omega /\left.d \phi\right|_{0}\right)}{3 a^{2} \phi_{(0)}}\left(\phi_{, \eta}^{(0)}\right)^{2} .
$$

In order to compute $h^{a b} \nabla_{a} \nabla_{b} \phi$, we need the Christoffel symbols

$$
\begin{aligned}
& \Gamma_{22}^{0}=\frac{a_{, \eta}}{a} r^{2}[1-2(\Phi+\psi)], \\
& \Gamma_{22}^{1}=-r+r^{2} \Phi_{, r}, \\
& \Gamma_{33}^{0}=\frac{a_{, \eta}}{a} r^{2} \sin ^{2} \theta(1-2 \Phi-2 \psi), \\
& \Gamma_{33}^{1}=r \sin ^{2} \theta\left(-1+r \Phi_{, r}\right),
\end{aligned}
$$


and the second covariant derivatives

$$
\begin{aligned}
& \nabla_{2} \nabla_{2} \phi=-\frac{a_{, \eta}}{a} r^{2} \phi_{, \eta}^{(0)}+\frac{2 a_{, \eta}}{a} r^{2} \phi_{, \eta}^{(0)}(\Phi+\psi)+r \delta \phi_{, r} \\
& \nabla_{3} \nabla_{3} \phi=-\frac{a_{, \eta}}{a} r^{2} \sin ^{2} \theta \phi_{, \eta}^{(0)}+\frac{2 a_{, \eta}}{a} r^{2} \sin ^{2} \theta \phi_{, \eta}^{(0)}+r^{2} \sin ^{2} \theta \delta \phi_{, r} .
\end{aligned}
$$

These expressions yield

$$
h^{a b} \nabla_{a} \nabla_{b} \phi=\frac{4 a_{, \eta}}{a^{3}}(\Phi+\psi) \phi_{, \eta}^{(0)}-\frac{4 a_{, \eta}}{a^{3}} \phi_{, \eta}^{(0)} \Phi-\frac{2 a_{, \eta}}{a^{3}} \phi_{, \eta}^{(0)}+\frac{2 \delta \phi_{, r}}{a^{2} r},
$$

while

$$
\frac{V}{3}=\frac{V\left(\phi_{(0)}+\delta \phi\right)}{3}=\frac{V_{0}+V_{0}^{\prime} \delta \phi}{3} .
$$

By inserting these expressions in Eq. (17), a few terms cancel out and one is left with

$$
\begin{aligned}
& M_{S T}=\frac{R^{3}}{4}\left[\frac{16 \pi}{3} \rho_{(0)}+\frac{\omega_{0}}{3 a^{2} \phi_{(0)}}\left(\phi_{, \eta}^{(0)}\right)^{2}-\frac{2 a_{, \eta}}{a^{3}} \phi_{, \eta}^{(0)}+\frac{V_{0}}{3}\right] \\
& +\frac{R_{(0)}^{3}}{4}\left\{\frac{2 \phi^{(0)} C_{2323}}{a^{4} r^{4} \sin ^{2} \theta}+\frac{16 \pi}{3}\left[-4\left(\rho_{(0)}+P_{(0)}\right) \psi+\delta \rho-2 P_{(0)} \Phi\right]+\frac{\left(-2 \omega_{0} \psi+\omega_{0}^{\prime} \delta \phi\right)}{3 a^{2} \phi_{(0)}}\left(\phi_{, \eta}^{(0)}\right)^{2}+\frac{4 a_{, \eta}}{a^{3}} \phi_{, \eta}^{(0)} \psi\right. \\
& \left.+\frac{2 \delta \phi_{, r}}{a^{2} r}+\frac{V_{0}^{\prime}}{3} \delta \phi\right\} .
\end{aligned}
$$

Using the unperturbed Friedmann equation of scalar-tensor gravity in a spatially flat FLRW universe

$$
H_{(0)}^{2}=\frac{8 \pi}{3 \phi_{(0)}} \rho_{(0)}+\frac{\omega}{6}\left(\frac{\dot{\phi}_{(0)}}{\phi_{(0)}}\right)^{2}-H \frac{\dot{\phi}_{(0)}}{\phi_{(0)}}+\frac{V_{0}}{6 \phi^{(0)}}
$$

(where an overdot denotes differentiation with respect to the comoving time $t$ of the background FLRW universe, related to the conformal time $\eta$ by $d t=a d \eta$ ), the first square bracket on the right hand side of Eq. (45) becomes $\frac{R^{3}}{4}[\ldots]=\frac{H_{(0)}^{2} R^{3} \phi_{(0)}}{2}$, so that

$$
\begin{aligned}
M_{S T}= & \frac{H_{(0)}^{2} R^{3} \phi_{(0)}}{2}+\frac{R_{(0)}^{3}}{4}\left\{\frac{2 \phi^{(0)} C_{2323}}{a^{4} r^{4} \sin ^{2} \theta}+\frac{16 \pi}{3}\left[-4\left(\rho_{(0)}+P_{(0)}\right) \psi+\delta \rho-2 P_{(0)} \Phi\right]+\frac{\left(-2 \omega_{0} \psi+\omega_{0}^{\prime} \delta \phi\right)}{3 a^{2} \phi_{(0)}}\left(\phi_{, \eta}^{(0)}\right)^{2}\right. \\
& \left.+\frac{4 a, \eta}{a^{3}} \phi_{, \eta}^{(0)} \psi+\frac{2 \delta \phi_{, r}}{a^{2} r}+\frac{V_{0}^{\prime}}{3} \delta \phi\right\}
\end{aligned}
$$

Now, using $R^{3}=R_{(0)}^{3}(1-3 \Phi)$ and $M_{S T}^{(0)}=H_{(0)}^{2} R_{(0)}^{3} \phi_{(0)} / 2$, one obtains

$$
\begin{aligned}
M_{S T}= & M_{S T}^{(0)}(1-3 \Phi)+\frac{R_{(0)}^{3} \phi_{(0)}}{4}\left\{\frac{2}{3 a^{2}}\left(\frac{\psi^{\prime}}{r}+\frac{\Phi^{\prime}}{r}-\psi^{\prime \prime}-\Phi^{\prime \prime}\right)\right. \\
& +\frac{16 \pi}{3}\left[-4\left(\rho_{(0)}+P_{(0)}\right) \psi+\delta \rho-2 P_{(0)} \Phi\right]+\frac{\left(-2 \omega_{0} \psi+\omega_{0}^{\prime} \delta \phi\right)}{3 a^{2} \phi_{(0)}}\left(\phi_{, \eta}^{(0)}\right)^{2} \\
& \left.+\frac{4 a_{, \eta}}{a^{3}} \phi_{, \eta}^{(0)} \psi+\frac{2 \delta \phi_{, r}}{a^{2} r}+\frac{V_{0}^{\prime}}{3} \delta \phi\right\} .
\end{aligned}
$$

The structures interesting for turnaround physics that have been studied in the literature are galaxy groups and clusters [19, 21 25]); they have size $\sim R$ much smaller than the Hubble radius $H^{-1}$ and, since $|\Phi| \ll 1$, we can simplify: $M_{S T}^{(0)}(1-3 \Phi)=\frac{H_{(0)}^{2} R_{(0)}^{3} \phi_{(0)}}{2}(1-3 \Phi) \approx \frac{H_{(0)}^{2} R_{(0)}^{3} \phi_{(0)}}{2}=M_{S T}^{(0)}$ and

$$
M_{S T} \simeq M_{S T}^{(0)}+\delta M_{S T} .
$$


According to the procedure of Ref. [35], the (comoving) turnaround radius is obtained by setting

$$
M_{S T}^{(0)}=\left|\delta M_{S T}\right|
$$

or

$$
\begin{aligned}
H_{(0)}^{2}= & \frac{2}{3 a^{2}}\left(\frac{\psi^{\prime}}{r}+\frac{\Phi^{\prime}}{r}-\psi^{\prime \prime}-\Phi^{\prime \prime}\right) \\
& +\frac{16 \pi}{3}\left[-4\left(\rho_{(0)}+P_{(0)}\right) \psi+\delta \rho-2 P_{(0)} \Phi\right] \\
& +\frac{\left(-2 \omega_{0} \psi+\omega_{0}^{\prime} \delta \phi\right)}{3 a^{2} \phi_{(0)}}\left(\phi_{, \eta}^{(0)}\right)^{2} \\
& +\frac{4 a_{, \eta}}{a^{3}} \phi_{, \eta}^{(0)} \psi+\frac{2 \delta \phi_{, r}}{a^{2} r}+\frac{V_{0}^{\prime}}{3} \delta \phi .
\end{aligned}
$$

This is the equation satisfied by the tunaround radius in scalar-tensor gravity.

\section{COMPARISON WITH PREVIOUS LITERATURE}

As a check of the previous result, consider the special case of GR in which FLRW space, sourced by dark energy with equation of state $P_{(0)}=w \rho_{(0)}$ and $w \simeq-1$, is perturbed by a point-like mass $m$ and

$$
\psi=\Phi=-\frac{m}{r}, \quad \phi=\text { const. }
$$

Equation (51) then reduces to

$$
H_{(0)}^{2}=\frac{2 m}{a^{2} r^{3}}+\frac{16 \pi}{3} \rho_{(0)}|3 w+1| \frac{m}{r} .
$$

The equations expressing the comoving and areal values of the turnaround radius of a spherical structure are

$$
\begin{gathered}
H_{(0)}^{2} r^{3}-2|3 w+2| H_{(0)}^{2} m r^{2}-\frac{2 m}{a^{2}}=0, \\
H_{(0)}^{2} R^{3}-2|3 w+2| H_{(0)}^{2}(m a) R^{2}-2(m a)=0,
\end{gathered}
$$

respectively. Since $m a \ll R$, one can neglect the term proportional to $m a R^{2}$ in comparison with $R^{3}$, obtaining the turnaround radius

$$
R=\left(\frac{2 m a}{H_{(0)}^{2}}\right)^{1 / 3}
$$

which reproduces the well known result for this case, corresponding to Eq. (2.7) of Ref. [35].

\section{AN EXAMPLE IN BRANS-DICKE GRAVITY}

As an example, we apply the formula derived in Sec. II to a family of inhomogeneous, time-dependent solutions of Brans-Dicke theory found in [42] and interpreted in [43]. These are exact solutions describing spherical inhomogeneities embedded in a FLRW universe sourced by a perfect fluid, but we will linearize them since only Newtonian-like perturbations of a FLRW background universe are of interest here.

The general family of solutions of Brans-Dicke theory found in [42] reads

$$
d s^{2}=a^{2}(\eta)\left[-A^{2 \alpha}(r) d \eta^{2}+B(r)\left(d r^{2}+r^{2} d \Omega_{(2)}^{2}\right)\right]
$$


where

$$
\begin{aligned}
A(r) & =\frac{1-m /(2 \alpha r)}{1+m /(2 \alpha r)}, \\
B(r) & =\left(1+\frac{m}{2 \alpha r}\right)^{4} A^{\frac{2(\alpha-1)(\alpha+2)}{\alpha}}, \\
a(t) & =a_{*}\left(\frac{t-t_{1}}{t_{0}}\right)^{\frac{2 \omega(2-\gamma)+2}{3 \omega \gamma(2-\gamma)+4}} \equiv a_{*} \tau^{\beta}, \\
\phi(t, r) & =\phi_{*} \tau^{\frac{2(4-3 \gamma)}{3 \omega(2-\gamma)+4}} A^{\frac{-2\left(\alpha^{2}-1\right)}{\alpha}}, \\
\rho(t, r) & =\rho_{*}\left(\frac{a_{*}}{a(t)}\right)^{3 \gamma} A^{-2 \alpha}, \\
\alpha & =\sqrt{\frac{2(\omega+2)}{2 \omega+3}},
\end{aligned}
$$

where $m$ is a mass parameter, $t_{0}, t_{1}, a_{*}, \phi_{*}$ and $\rho_{*}$ are constants, the comoving time $t$ of the "background" is related to the conformal time $\eta$ by $d t=a d \eta$ and we introduced $\beta \equiv \frac{2 \omega(2-\gamma)+2}{3 \omega \gamma(2-\gamma)+4}, \tau \equiv \frac{t-t_{1}}{t_{0}}$. The energy density $\rho$ and pressure $P$ of the cosmic fluid obey the equation of state $P=(\gamma-1) \rho$ with $\gamma=$ const., the Brans-Dicke parameter $\omega$ is constant, and there is no potential $V$ for the Brans-Dicke scalar field $\phi$. The parameter $\alpha$ is real if $\omega<-2$ or if $\omega>-3 / 2$.

Since we are interested in a universe with dust in modified gravity, we set $\gamma=1$, then $P_{(0)}=0$ and $\rho_{(0)}(t) \simeq a^{-3}$. The universe approaches a Big Rip if the exponent of the scale factor $\frac{2(\omega+1)}{3 \omega+4}<0$, which is satisfied in the range $-4 / 3<\omega<-1$ which we adopt. Since here the Brans-Dicke scalar is massless, this range is not realistic because it violates the Cassini bound $|\omega|>50000$. Nevertheless, we use this solution as an example because there are very few inomogeneous solutions of modified gravity in the literature and here we want to impose the extra constraints that the background FLRW universe is accelerated and is sourced by a dust.

We now linearize the exact solution of [42] for $m / r \ll 1$, obtaining

$$
\begin{aligned}
A(r) & \simeq 1-\frac{m}{\alpha r} \\
B(r) & \simeq 1-\frac{2 m\left(\alpha^{2}-2\right)}{\alpha^{2} r}, \\
\phi(t, r) & =\phi_{*} \tau^{\frac{2}{3 \omega+4}}\left[1+\frac{2 m}{r}\left(\frac{\alpha^{2}-1}{\alpha^{2}}\right)\right] .
\end{aligned}
$$

By comparing the linearized line elements (7) and (57), one obtains

$$
\begin{aligned}
& \psi(r)=-\frac{m}{r}, \\
& \Phi(r)=\frac{\left(\alpha^{2}-2\right) m}{\alpha^{2} r},
\end{aligned}
$$

while, for short observation times, one has

$$
\begin{aligned}
& \phi_{(0)}(t)=\phi_{*} \tau^{\frac{2}{3 \omega+4}}, \quad \delta \phi=\phi_{(0)}(t) \frac{2 m}{r}\left(\frac{\alpha^{2}-1}{\alpha^{2}}\right) \simeq \phi_{(0)}(\text { now }) \frac{2 m}{r}\left(\frac{\alpha^{2}-1}{\alpha^{2}}\right), \\
& \rho_{(0)}(t)=\frac{\rho_{*}}{\tau^{3 \beta}}, \quad \delta \rho=\rho_{(0)}(t) \frac{2 m}{r} \simeq \rho_{(0)}(\text { now }) \frac{2 m}{r}
\end{aligned}
$$

Taking advantage of Eq. (60) one finds

$$
H_{(0)}=\frac{\beta}{t_{0} \tau}
$$


and that Eqs. (67) and (68) suggest

$$
\frac{\left(\psi^{\prime}+\Phi^{\prime}\right)}{r}-\left(\psi^{\prime \prime}+\Phi^{\prime \prime}\right)=\frac{6 m}{\alpha^{2} r^{3}}
$$

Then, Eq. (51) locating the turnaround radius becomes the cubic equation

$$
\alpha_{0}(t) r^{3}+\alpha_{1}(t) r^{2}+\alpha_{2}(t)=0
$$

where the coefficients are time-dependent, expressing the fact that the turnaround radius evolves with time, and are

$$
\begin{gathered}
\alpha_{0}(t)=\left(\frac{\beta}{t_{0} \tau}\right)^{2}, \\
\alpha_{1}(t)=-2 m\left[\frac{16 \pi}{3}\left(\frac{2 \rho_{*}}{\tau^{3 \beta}}+\rho_{(0)}(\text { now })\right)-\frac{4 \phi_{*}(5 \omega+6)}{3 t_{0}^{2}(3 \omega+4)^{2}} \tau^{-\frac{6(\omega+1)}{3 \omega+4}}\right], \\
\alpha_{2}(t)=-\frac{4 m}{a_{*}^{2} \tau^{2 \beta} \alpha^{2}}\left[1-\left(\alpha^{2}-1\right) \phi_{(0)}(\text { now })\right] .
\end{gathered}
$$

\section{CONCLUSIONS}

The turnaround radius in scalar-tensor gravity is now derived using the new definition (50) already applied to GR in [35] to obtain a gauge-invariant expression of the turnaround radius. This definition differs from another one based on the study of radial timelike geodesics. However, in GR, the final values of the turnaround radius obtained with these two different definitions do not differ by much [35], and the observational error in the determination of the turnaround radius of realistic structures in the sky is going to be more significant than this small difference between different definitions. However, the theory needs to be put on a sound basis before the significant challenges posed by the observational determination of the turnaround radius can be addressed. The new definition of scalar-tensor mass generalizing the Hawking-Hayward construct to scalar-tensor gravity [37] produces Eq. (51) which locates the turnaround radius in this class of theories. In general, this equation appears more complicated than the corresponding one of GR due to the presence of the extra scalar degree of freedom. There will certainly be simplifications to this formula in particular scalar-tensor scenarios which include an accelerated universe at the present epoch, to which we do not want to commit at the moment in order to preserve generality. The application of the general formula (51) to detailed scalar-tensor models will be considered elsewhere.

\section{Acknowledgments}

We are grateful to Antonio Enea Romano for a discussion. This work is supported, in part, by the Natural Science and Engineering Research Council of Canada (Grant No. 2016-03803 to V.F.), by Bishops University, and by the Perimeter Institute for Theoretical Physics. Research at Perimeter Institute is supported by the Government of Canada through Industry Canada and by the Province of Ontario through the Ministry of Economic Development and Innovation. The work of AG has been carried out in the framework of the activities of the Italian National Group for Mathematical Physics [Gruppo Nazionale per la Fisica Matematica (GNFM), Istituto Nazionale di Alta Matematica (INdAM)]. 
[1] S. Perlmutter et al. [Supernova Cosmology Project Collaboration], Nature 391, 51 (1998).

[2] S. Perlmutter et al. [Supernova Cosmology Project Collaboration], Astrophys. J. 517, 565 (1999).

[3] M.T. Busha, F.C. Adams, R.H. Wechsler, and A.E. Evrard, Astrophys. J. 596, 713 (2003).

[4] V. Pavlidou and T.N. Tomaras, J. Cosmol. Astropart. Phys. 1409, 020 (2014).

[5] V. Pavlidou, N. Tetradis, and T.N. Tomaras, JCAP 1405, 017 (2014).

6] S. Bhattacharya and T.N. Tomaras, Eur. Phys. J. C 77, 526 (2017).

[7] M. Cataneo and D. Rapetti, Int. J. Mod. Phys. D 27, 1848006 (2018).

[8] Z. Roupas, Universe 5, 12 (2019).

[9] V. Faraoni, Phys. Dark Universe 11, 11 (2016).

[10] S. Bhattacharya, K.F. Dialektopoulos, and T.N. Tomaras, J. Cosmol. Astropart. Phys. 1605, 036 (2016).

[11] S. Bhattacharya, K.F. Dialektopoulos, A.E. Romano, C. Skordis, and T.N. Tomaras, J. Cosmol. Astropart. Phys. 1707, 018 (2017).

[12] S. Nojiri, S.D. Odintsov, and V. Faraoni, Phys. Rev. D 98, 024005 (2018).

[13] S. Capozziello, K.F. Dialektopoulos, and O. Luongo, Int. J. Mod. Phys. D 28, 1950058 (2018).

[14] R.C.C. Lopes, R. Voivodic, L.R. Abramo and L. Sodré, J. Cosmol. Astropart. Phys. 1809, 010 (2018).

[15] R. C. C. Lopes, R. Voivodic, L. R. Abramo, and L. Sodré, J. Cosmol. Astropart. Phys. 1907, 026 (2019).

[16] J.D. Barrow and J. Silk, Astrophys. J. 250, 432 (1981); J.D. Barrow and P. Saich, Mon. Not. Roy. Astron. Soc. 262, 717 (1993); J.D. Barrow and G. Götz, Class. Quantum Grav. 6, 1253 (1989).

[17] A. Giusti and V. Faraoni, Phys. Dark Universe 26, 100353 (2019).

[18] A. Giusti and V. Faraoni, arXiv:1911.05130.

[19] S.H. Hansen, F. Hassani, L. Lombriser, and M. Kunz, JCAP 2001, 048 (2020).

[20] S. Bhattacharya and T.N. Tomaras, arXiv:1911.06228.

[21] G. Korkidis, V. Pavlidou, K. Tassis, E. Ntormousi, T.N. Tomaras, and K. Kovlakas, arXiv:1912.08216.

[22] J. Lee, S. Kim, and S.C. Rey, Astrophys. J. 815, 43 (2015).

[23] J. Lee and G. Yepes, Astrophys. J. 832, 185 (2016).

[24] J. Lee, Astrophys. J. 856, 57 (2018).

[25] J. Lee, Astrophys. J. 832, 123 (2016).

[26] S. Hawking, J. Math. Phys. (N.Y.) 9, 598 (1968).

[27] S.A. Hayward, Phys. Rev. D 49, 831 (1994).

[28] S.A. Hayward, Phys. Rev. D 53, 1938 (1996).

[29] C.W. Misner and D.H. Sharp, Phys. Rev. 136, B571 (1964); W.C. Hernandez and C.W. Misner, Astrophys. J. 143, 452 (1966).

[30] A. Prain, V. Vitagliano, V. Faraoni, and M. Lapierre-Léonard, Classical Quantum Grav. 33, 145008 (2016).

[31] V. Faraoni, M. Lapierre-Léonard, and A. Prain, Phys. Rev. D 92, 023511 (2015).

[32] V. Faraoni and V. Vitagliano, Phys. Rev. D 89, 064015 (2014).

[33] V. Faraoni and M. Lapierre-Léonard, Phys. Rev. D 95, 023509 (2017).

[34] M. Lapierre-Léonard, V. Faraoni, and F. Hammad, Phys. Rev. D 96, 083525 (2017).

[35] V. Faraoni, M. Lapierre-Léonard, and A. Prain, J. Cosmol. Astropart. Phys. 10, 013 (2015).

[36] R.-G. Cai, L.M. Cao, Y.P. Hu, and N. Ohta, Phys. Rev. D 80, 104016 (2009); R.-G. Cai, L.M. Cao, Y.P. Hu, and S.P. Kim, Phys. Rev. D 78, 124012 (2008); H. Zhang, Y. Hu, and X.Z. Li, Phys. Rev. D 90, 024062 (2014); S.-F. Wu, B. Wang, and G.-H. Yang, Nucl. Phys. B 799, 330 (2008); G. Cognola, O. Gorbunova, L. Sebastiani, and S. Zerbini, Phys. Rev. D 84, 023515 (2011); F. Hammad, Classical Quantum Grav. 33, 235016 (2016); Int. J. Mod. Phys. D 25, 1650081 (2016).

[37] V. Faraoni, Classical Quantum Grav. 33, 015007 (2015); V. Faraoni and J. Côté, Phys. Rev. D 100, 084015 (2019).

[38] J.C. Hwang, Phys. Rev. D 42, 2601 (1990); J.C. Hwang, Class. Quantum Grav. 7, 1613 (1990); J.C. Hwang, Class. Quantum Grav. 8, 195 (1991); J.C. Hwang, Class. Quantum Grav. 14, 3327 (1997); J.C. Hwang H. and Noh, Phys. Rev. D 54, 1460 (1996).

[39] M. Salgado, Class. Quantum Grav. 23, 4719 (2006).

[40] R.M. Wald, General Relativity (Chicago University Press, Chicago, 1984).

[41] T.P. Sotiriou and V. Faraoni, Rev. Mod. Phys. 82, 451 (2010); A. De Felice and S. Tsujikawa, Living Rev. Relat. 13, 3 (2010); S. Nojiri and S.D. Odintsov, Phys. Rept. 505, 59 (2011).

[42] T. Clifton, D.F. Mota, and J.D. Barrow, Mont. Not. Roy. Astron. Soc. 358, 601 (2005).

[43] V. Faraoni, V. Vitagliano, T.P. Sotiriou, and S. Liberati, Phys. Rev. D 86, 064040 (2012). 